\title{
LHC SUSY and WIMP dark matter searches confront the string theory landscape
}

\author{
Howard Baer, ${ }^{a}$ Vernon Barger, ${ }^{b}$ Shadman Salam, ${ }^{a}$ Hasan Serce ${ }^{b}$ and Kuver Sinha ${ }^{a}$ \\ ${ }^{a}$ Homer L. Dodge Department of Physics and Astronomy, University of Oklahoma, \\ 440 West Brooks, Norman, OK 73019, U.S.A. \\ ${ }^{b}$ Department of Physics, University of Wisconsin, \\ 1150 University Avenue, Madison, WI 53706 U.S.A. \\ E-mail: baer@ou.edu, barger@pheno.wisc.edu, shadman.salam@ou.edu, \\ serce@ou.edu, kuver.sinha@ou.edu
}

ABSTRACT: The string theory landscape of vacua solutions provides physicists with some understanding as to the magnitude of the cosmological constant. Similar reasoning can be applied to the magnitude of the soft SUSY breaking terms in supersymmetric models of particle physics: there appears to be a statistical draw towards large soft terms which is tempered by the anthropic requirement of the weak scale lying not too far from $\sim 100 \mathrm{GeV}$. For a mild statistical draw of $m_{\text {soft }}^{n}$ with $n=1$ (as expected from SUSY breaking due to a single $F$ term) then the light Higgs mass is preferred at $\sim 125 \mathrm{GeV}$ while sparticles are all pulled beyond LHC bounds. We confront a variety of LHC and WIMP dark matter search limits with the statistical expectations from a fertile patch of string theory landscape. The end result is that LHC and WIMP dark matter detectors see exactly that which is expected from the landscape: a Standard Model-like Higgs boson of mass $125 \mathrm{GeV}$ but as yet no sign of sparticles or WIMP dark matter. SUSY from the $n=1$ landscape is most likely to emerge at LHC in the soft opposite-sign dilepton plus jet plus MET channel. Multi-ton noble liquid WIMP detectors should be able to completely explore the $n=1$ landscape parameter space.

KEYWORDs: Supersymmetry Phenomenology, Strings and branes phenomenology

ARXIV EPrint: 1901.11060 


\section{Contents}

1 Introduction 1

2 Statistics of the SUSY breaking scale 3

2.1 Brief review of some previous work and goals of the present work 6

3 Landscape predictions vs. LHC search limits $\quad 8$

3.1 Landscape predictions for NUHM2 model 8

3.2 Landscape predictions for NUHM3 model 9

4 Landscape predictions vs. WIMP DM search limits 13

$\begin{array}{llr}5 & \text { Conclusions } & 16\end{array}$

\section{Introduction}

It is sometimes lamented that the emergence of the landscape of string vacua [1-3] has rendered string theory non-predictive since how are we to pick out the (meta-stable) vacuum corresponding to our universe from (perhaps) of order $10^{500}$ possibilities? Such sentiment ignores one of the great predictions of the latter 20th century $[4,5]$ : namely that given a multiverse which includes a vast assortment of pocket-universes with varying cosmological constants, then it may not be surprising to find $\Lambda \sim 10^{-120} m_{P}^{4}$ since if it was much bigger, then galaxy condensation would not occur and we would not even be here to discuss the issue. The situation is portrayed in figure 1 which depicts the fact that the cosmological constant ought to be at its most natural value subject to the constraint that we can exist to observe it. Such anthropic reasoning relies on the existence of a vast landscape of possibilities that is provided for by the discretuum of flux vacua from string theory $[1-3,6,7]$.

Can such reasoning be applied to the origin of other mass scales that appear in fundamental physics? ${ }^{1}$ An obvious target would be the magnitude of the weak scale $\left(m_{\text {weak }} \simeq m_{W, Z, h} \simeq 100 \mathrm{GeV}\right)$ and why it is suppressed by 16 orders of magnitude compared to the (reduced) Planck scale $m_{P} \simeq 2.4 \times 10^{18} \mathrm{GeV}$. Anthropically, one expects a universe with electroweak symmetry properly broken such that weak bosons gain mass $m_{W, Z} \sim 100 \mathrm{GeV}$ while solutions with charge or color breaking minima would be excluded. In addition, nuclear physics calculations by Agrawal et al. $[9,10]$ require that the magnitude of the weak scale shouldn't exceed its measured value by a factor of $2-3$ in order to gain a livable universe.

\footnotetext{
${ }^{1}$ Weinberg states [8]: "The most optimistic hypothesis is that the only constants that scan are the few whose dimensionality is a positive power of mass: the vacuum energy, and whatever scalar mass or masses set the scale of electroweak symmetry breaking".
} 


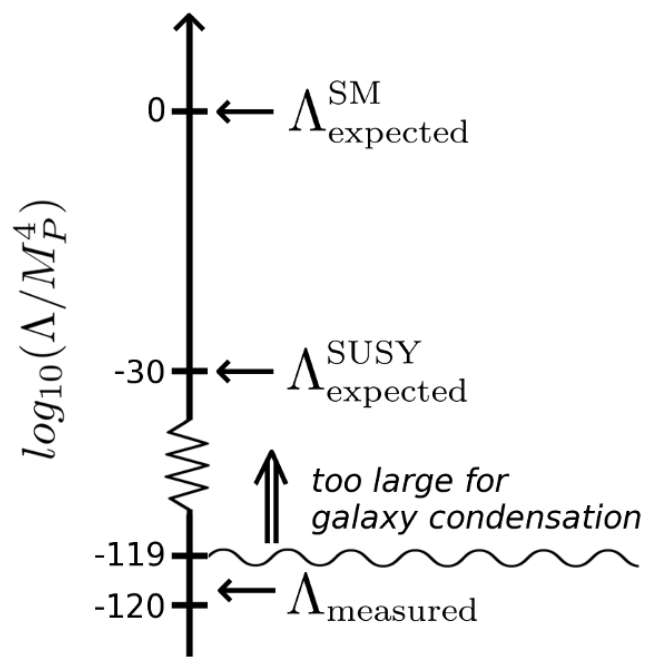

Figure 1. Log portrayal of expected parameter space of the cosmological constant $\Lambda$ from the string theory landscape.

Given that quantum corrections to the Higgs mass diverge quadratically with the theory cutoff $\Lambda_{\mathrm{SM}}$, it seems the Standard Model (SM) with $\Lambda_{\mathrm{SM}} \gg m_{\text {weak }}$ would be a rare occurrance within the landscape since one would be required to select only those vacuum solutions with highly fine-tuned scan parameters. In a landscape containing both SM and supersymmetric SM solutions (SSM), one would expect vastly more SSM solutions with $m_{\text {weak }} \simeq 100 \mathrm{GeV}$ since then quantum corrections to $m_{h}$ diverge only logarithmically.

In fact, this discussion brings up the issue of how naturalness is connected to vacua selection in the multiverse. Here we adopt the notion of practical naturalness:

An observable $\mathcal{O} \equiv o_{1}+\cdots+o_{n}$ is natural if all independent contributions $o_{i}$ to $\mathcal{O}$ are comparable to or less than $\mathcal{O}$.

This is because if any $o_{i} \gg \mathcal{O}$, then some other contribution $o_{j}(j \neq i)$ would have to be fine-tuned to a large opposite-sign value to keep $\mathcal{O}$ at its measured value. The notion of practical naturalness may be compared with what Douglas calls stringy naturalness [11]:

An effective field theory (or specific coupling or observable) $T_{1}$ is more natural in string theory than $T_{2}$ if the number of phenomenologically acceptable vacua leading to $T_{1}$ is larger than the number leading to $T_{2}$.

In a landscape of vacua where independent contributions $o_{i}$ to observable $\mathcal{O}$ are uniformly distributed, then it follows that many more vacua are likely to exist where the $o_{i}$ are comparable to $\mathcal{O}$ than where some $o_{i} \gg \mathcal{O}$ so that some other value $o_{j \neq i} \simeq-o_{i}$. Thus, we expect these two definitions to be equivalent descriptions of naturalness. The landscape, if it is to be predictive, is predictive in the statistical sense: the more prevalent solutions are statistically more likely. This gives the connection between landscape statistics and 
naturalness: vacua with natural observables are expected to be far more common than vacua with unnatural observables. ${ }^{2}$

Thus, in this paper we will focus on vacua solutions which include the Minimal Supersymmetric Standard Model (MSSM) as their weak scale effective theory. This restricts our attention to a fertile patch of landscape solutions which include the SM as the weak scale effective theory (in accord with experiment) but where the weak scale is stable against quantum corrections (as in the MSSM). We will further assume that the MSSM arises from a 4-d supergravity theory (SUGRA) where SUSY breaking occurs via spontaneous SUGRA breaking in a hidden sector of the theory with a, perhaps, complicated SUSY breaking sector including possibly numerous SUSY breaking $F$ - and $D$-term fields which gain vevs. The question that can be addressed is then: what does a statistical sampling of this fertile patch of the landscape say about the scale of SUSY breaking, and hence the likelihood of observable signals at the CERN Large Hadron Collider (LHC) or at WIMP dark matter direct and indirect detection experiments? Indeed, this question has already been investigated early on by Douglas et al. [13-15], Susskind [12] and by Dine et al. [16, 17]. (For some reviews, see e.g. refs. [18, 19].)

\section{Statistics of the SUSY breaking scale}

In this section, we assume a vast ensemble of string vacua states which give rise to a $D=4$, $N=1$ supergravity effective field theory at high energies. Furthermore, the theory consists of a visible sector containing the MSSM along with a perhaps large assortment of fields that comprise the hidden sector. The scalar potential is given by the usual supergravity form $[20]$

$$
\begin{aligned}
V & =e^{K / m_{P}^{2}}\left(g^{i \bar{j}} D_{i} W D_{\bar{j}} W^{*}-\frac{3}{m_{P}^{2}}|W|^{2}\right)+\frac{1}{2} \sum_{\alpha} D_{\alpha}^{2} \\
& =e^{K / m_{P}^{2}}\left(\sum_{i}\left|F_{i}\right|^{2}-3 \frac{|W|^{2}}{m_{P}^{2}}\right)+\frac{1}{2} \sum_{\alpha} D_{\alpha}^{2}
\end{aligned}
$$

where $W$ is the holomorphic superpotential, $K$ is the real Kähler potential and $F_{i}=D_{i} W=$ $D W / D \phi^{i} \equiv \partial W / \partial \phi^{i}+\left(1 / m_{P}^{2}\right)\left(\partial K / \partial \phi^{i}\right) W$ are the $F$-terms and $D_{\alpha} \sim \sum \phi^{\dagger} g t_{\alpha} \phi$ are the $D$-terms and the $\phi^{i}$ are chiral superfields. Supergravity is assumed to be broken spontaneously via the super-Higgs mechanism either via $F$-type breaking or $D$-type breaking or in general a combination of both leading to a gravitino mass $m_{3 / 2}=e^{K / 2 m_{P}^{2}}|W| / m_{P}^{2}$. The (metastable) minima of the scalar potential can be found by requiring $\partial V / \partial \phi^{i}=0$ with $\partial^{2} V / \partial \phi^{i} \partial \phi^{j}>0$ to ensure a local minimum. The cosmological constant is given by

$$
\Lambda_{c c}=m_{\text {hidden }}^{4}-3 e^{K / m_{P}^{2}}|W|^{2} / m_{P}^{2}
$$

\footnotetext{
${ }^{2}$ Since the landscape allows for an apparently unnatural value of $\Lambda_{c c} \ll m_{P}^{4}$, it is sometimes interpreted that vacua with other highly unnatural observables should also be entertained. In the case of $\Lambda_{c c}$, we should find ourselves in a universe where the cosmological constant is as natural as possible such that we gain a livable universe. Then we would expect $\Lambda_{c c} \sim 10^{-120} m_{P}^{4}$ rather than say $10^{-200} m_{P}^{4}$.
} 
where $m_{\text {hidden }}^{4}=\sum_{i}\left|F_{i}\right|^{2}+\frac{1}{2} \sum_{\alpha} D_{\alpha}^{2}$ is a mass scale associated with the hidden sector (and usually in SUGRA-mediated models it is assumed $m_{\text {hidden }} \sim 10^{12} \mathrm{GeV}$ such that the gravitino gets a mass $\left.m_{3 / 2} \sim m_{\text {hidden }}^{2} / m_{P}\right)$.

According to Douglas et al. [14] from investigations of flux compactifications in IIB string theory, the distribution of vacua ought to have the form

$$
d N_{\text {vac }}\left[m_{\text {hidden }}^{2}, m_{\text {weak }}, \Lambda\right]=f_{\mathrm{SUSY}}\left(m_{\text {hidden }}^{2}\right) \cdot f_{\text {EWFT }} \cdot f_{c c} \cdot d m_{\text {hidden }}^{2}
$$

where we define the weak scale $m_{\text {weak }} \simeq m_{W, Z, h} \simeq 100 \mathrm{GeV}$ and where $m_{\text {hidden }}$ sets the scale for SUSY breaking with $m_{\text {hidden }}^{2}=\sum_{i}\left|F_{i}\right|^{2}+\frac{1}{2} \sum_{\alpha} D_{\alpha}^{2}$ for a (in general) more complicated SUSY breaking sector containing multiple sources of SUSY breaking, as may be expected to occur in string theory.

The function $f_{\mathrm{SUSY}}$ contains the expected statistical distribution of SUSY breaking scales. This is related to the mass scale of MSSM soft terms as $m_{\text {soft }} \simeq m_{\text {hidden }}^{2} / m_{P}$. If the sources of SUSY breaking have uniformly distributed vacuum expectation values (vevs), then it is surmised that

$$
f_{\mathrm{SUSY}}\left(m_{\text {hidden }}^{2}\right) \sim\left(m_{\text {hidden }}^{2}\right)^{2 n_{F}+n_{D}-1}
$$

where $n_{F}$ is the number of $F$-breaking fields and $n_{D}$ is the number of $D$-term breaking fields in the hidden sector [11-14]. We will denote the collective exponent in $f_{\text {SUSY }}$ as $n \equiv 2 n_{F}+n_{D}-1$. Since the $F$ terms are complex-valued but the modulus $|F|$ sets the scale of SUSY breaking, then they contribute as $\left(m_{\text {hidden }}^{2}\right)^{2 n_{F}}$ whereas the real valued $D$ terms contribute as $\left(m_{\text {hidden }}^{2}\right)^{n_{D}}$. In terms of MSSM soft SUSY breaking parameters, one would expect a statistically uniform distribution of soft terms $m_{\text {soft }}^{0}$ only for a single $D$ term breaking field so that $n_{D}=1$. A single $F$-term breaking field leads to $f_{\mathrm{SUSY}} \sim m_{\text {soft }}^{1}$ so that there is a linearly increasing preference for large soft terms. For more complex configurations with larger number of $n_{F}$ and $n_{D}$, then there is an even greater statistical preference for large soft terms which could lead to a preference for models with high scale SUSY breaking.

Regarding the role of the cosmological constant in determining the SUSY breaking scale, a key observation of Denef and Douglas [13, 14] and Susskind [12] was that $W$ at the minima is distributed uniformly as a complex variable, and the distribution of $e^{K / m_{P}^{2}}|W|^{2} / m_{P}^{2}$ is not correlated with the distributions of $F_{i}$ and $D_{\alpha}$. Setting the cosmological constant to nearly zero, then, has no effect on the distribution of supersymmetry breaking scales. Physically, this can be understood by the fact that the superpotential receives contributions from many sectors of the theory, supersymmetric as well as nonsupersymmetric. The cosmological fine-tuning penalty is $f_{c c} \sim \Lambda / m^{4}$ where the above discussion leads to $m^{4} \sim m_{\text {string }}^{4}$ rather than $m^{4} \sim m_{\text {hidden }}^{4}$, rendering this term inconsequential for determining the number of vacua with a given SUSY breaking scale.

The final term $f_{\text {EWFT }}$ merits some discussion. Following ref. [21], an initial guess [12, $14,16,17]$ for $f_{\mathrm{EWFT}}$ was that $f_{\mathrm{EWFT}} \sim m_{\text {weak }}^{2} / m_{\text {soft }}^{2}$ which may be interpretted as conventional naturalness in that the larger the Little Hierarchy between $m_{\text {weak }}$ and $m_{\text {soft }}$, then the greater is the fine-tuning penalty. As pointed out in ref. [22], there are several problems with this ansatz. 
1. As soft terms such as the trilinear $A_{t}$ terms increase, one is ultimately forced into charge-or-color-breaking vacua of the MSSM $[23,24]$. These sorts of vacua must be entirely vetoed on anthropic grounds.

2. As high-scale soft terms such as $m_{H_{u}}^{2}$ increase too much, then they are no longer driven to negative values and electroweak symmetry isn't even broken. These nonEWSB solutions also should be vetoed on anthropic grounds.

3. As the high scale soft term $m_{H_{u}}^{2}$ increases, its weak scale value actually becomes smaller and smaller until EWSB is barely broken [25, 26]. This means the weak scale value of $m_{H_{u}}^{2}$ becomes more natural - a phenomena known as radiatively driven naturalness (RNS) [27, 28].

4. As the soft term $A_{t}$ increases, then cancellations can occur in the $\Sigma_{u}^{u}\left(\tilde{t}_{1,2}\right)$ contributions to the weak scale scalar potential, rendering their contributions closer to, not further from, the weak scale whilst at the same time lifting up the Higgs mass $m_{h}$ to the $125 \mathrm{GeV}$ range.

5. Even in the event of appropriate EWSB, the factor $f_{\mathrm{EWFT}} \sim m_{\mathrm{weak}}^{2} / m_{\mathrm{soft}}^{2}$ penalizes but does not forbid vacua with a weak scale far larger than its measured value. In contrast, Agrawal et al. [9, 10] have shown that a weak scale larger than $\sim 3$ times its measured value would lead to much weaker weak interactions and a disruption in nuclear synthesis reactions, and likely an unlivable universe as we know it. In addition, Susskind posits that an increased weak scale would lead to larger SM particle masses and consequent disruptions in both atomic and nuclear physics. From these calculations, it seems reasonable to veto SM-like vacua which lead to a weak scale more than (conservatively) four times its measured value.

To account for these issues, in ref. [22] the alternative of

$$
f_{\mathrm{EWFT}} \sim \Theta\left(30-\Delta_{\mathrm{EW}}\right)
$$

was suggested where $\Delta_{\mathrm{EW}}$ is the electroweak fine-tuning measure which just requires that weak scale contributions to $m_{Z}^{2}$ should be comparable to or less than $m_{Z}^{2}$. From the minimization conditions for the MSSM Higgs potential [29] one finds

$$
\frac{m_{Z}^{2}}{2}=\frac{m_{H_{d}}^{2}+\Sigma_{d}^{d}-\left(m_{H_{u}}^{2}+\Sigma_{u}^{u}\right) \tan ^{2} \beta}{\tan ^{2} \beta-1}-\mu^{2} \simeq-m_{H_{u}}^{2}-\Sigma_{u}^{u}-\mu^{2} .
$$

The naturalness measure $\Delta_{\mathrm{EW}}$ compares the largest contribution on the right-hand-side of eq. (2.7) to the value of $m_{Z}^{2} / 2$. The radiative corrections $\Sigma_{u}^{u}$ and $\Sigma_{d}^{d}$ include contributions from various particles and sparticles with sizeable Yukawa and/or gauge couplings to the Higgs sector. Usually the most important of these are

$$
\Sigma_{u}^{u}\left(\tilde{t}_{1,2}\right)=\frac{3}{16 \pi^{2}} F\left(m_{\tilde{t}_{1,2}}^{2}\right)\left[f_{t}^{2}-g_{Z}^{2} \mp \frac{f_{t}^{2} A_{t}^{2}-8 g_{Z}^{2}\left(\frac{1}{4}-\frac{2}{3} x_{W}\right) \Delta_{t}}{m_{\tilde{t}_{2}}^{2}-m_{\tilde{t}_{1}}^{2}}\right]
$$


where $f_{t}$ is the top-quark Yukawa coupling, $\Delta_{t}=\left(m_{\tilde{t}_{L}}^{2}-m_{\tilde{t}_{R}}^{2}\right) / 2+M_{Z}^{2} \cos 2 \beta\left(\frac{1}{4}-\frac{2}{3} x_{W}\right)$, $x_{W} \equiv \sin ^{2} \theta_{W}, F\left(m^{2}\right)=m^{2}\left(\log \frac{m^{2}}{Q^{2}}-1\right)$ and the optimized scale choice for evaluation of these corrections is $Q^{2}=m_{\tilde{t}_{1}} m_{\tilde{t}_{2}}$. In the denominator of eq. (2.8), the tree level expressions of $m_{\tilde{t}_{1,2}}^{2}$ should be used. Expressions for the remaining $\Sigma_{u}^{u}$ and $\Sigma_{d}^{d}$ terms are given in the appendix of ref. [28].

In the remainder of this paper, we will assume a solution to the SUSY $\mu$ problem such as the gravity-safe, electroweak natural axionic hybrid CCK model based on a $\mathbb{Z}_{24}^{R}$ symmetry in ref. [30]. As such, we invert the usual usage of $\Delta_{\mathrm{EW}}$ with fluid soft terms and $\mu$ term: instead, the weak scale (as typified by the value of $m_{Z}$ ) is no longer fixed at its measured value but is instead determined by eq. (2.7). In this case, values of $\Delta_{\mathrm{EW}} \gtrsim 30$ correspond to a value of $m_{\text {weak }} \gtrsim$ four times its measured value (in our sub-universe). The $\Theta$ function in eq. (2.6) guarantees that we veto vacua with CCB minima or no EWSB. It also vetoes properly broken Higgs potentials but where the weak scale is generated at more than four times its measured value.

\subsection{Brief review of some previous work and goals of the present work}

In ref. [26], an approach similar to Weinberg's anthropic solution to the cosmological constant was applied to determination of the SUSY breaking scale. It was assumed that there was a mild draw of the landscape towards large soft terms which was tempered by the anthropic requirement of a value for the weak scale which was not too far from its measured value by a factor $\sim$ four. The draw of $m_{H_{u}}^{2}$ towards large values, tempered by an appropriate breakdown of EW symmetry, led to barely-broken EW symmetry. This is the same as the naturalness condition that $m_{H_{u}}^{2}$ is driven to small negative values at the weak scale, and so gave a mechanism for why $m_{H_{u}}^{2}$ should be driven to natural values. It was also emphasized that the statistical draw to large soft terms must avoid CCB and no EWSB vacua while at the same time drawing towards a weak scale not too far from its measured value. The combined statistical/anthropic draw would pull soft terms towards a region where $A_{t}$ is large (but not too large) and where $m_{h} \sim 125 \mathrm{GeV}$.

In ref. [22], previous work by DD was adopted wherein soft SUSY breaking terms were actually selected according to $f_{\mathrm{SUSY}} \sim m_{\text {soft }}^{n}$ with $n=2 n_{F}+n_{D}-1$ while $f_{\text {EWFT }}$ was adopted as in eq. (2.6). This allowed for landscape probability distributions to be calculated for a host of superparticle and Higgs masses. Calculations were performed using the threeextra parameter non-universal Higgs model (NUHM3) with parameter space given by

$$
m_{0}(1,2), m_{0}(3), m_{1 / 2}, A_{0}, \tan \beta, \mu, m_{A} \text { (NUHM3) }
$$

where separate first/second and third generation soft scalar masses and a negative $A_{0}$ term were used. For $n=1$ or 2 , then the differential probability distribution for the Higgs mass $d P / d m_{h}$ acquired a firm peak around $m_{h} \sim 125 \mathrm{GeV}$.

Results from a scan over soft terms with $\mu: 100-360 \mathrm{GeV}$ with $A_{0}<0$ and $n=1$ (corresponding to a single SUSY breaking field $F$ ) are shown in figure 2 for the case where the generated weak scale is less than a factor four from its measured value. The green histogram shows results when LHC search constraints (except Higgs mass) are also imposed (see below). 


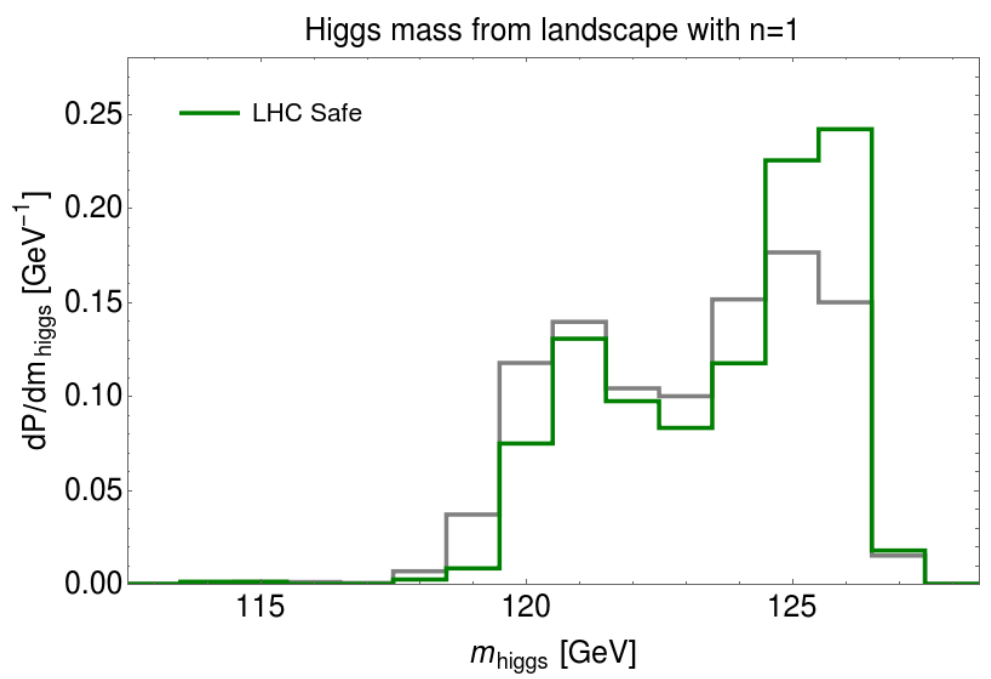

Figure 2. Statistical expectation for the mass of the Higgs boson from the string theory landscape which scans over single $F$-term SUSY breaking. The green histogram includes only LHC Run 2 safe points.

Furthermore, the probability distributions for other sparticle masses gave

- $m_{\tilde{g}} \sim 4 \pm 2 \mathrm{TeV}$,

- $m_{\tilde{t}_{1}} \sim 1.5 \pm 0.5 \mathrm{TeV}$,

- $m_{A} \sim 3 \pm 2 \mathrm{TeV}$,

- $\tan \beta \sim 13 \pm 7$,

- $m_{\widetilde{\chi}_{1}^{ \pm}, \widetilde{\chi}_{1,2}^{0}} \sim 200 \pm 100 \mathrm{GeV}$,

- $m_{\widetilde{\chi}_{2}^{0}}-m_{\widetilde{\chi}_{1}^{0}} \sim 7 \pm 3 \mathrm{GeV}$ and

- $m_{\tilde{q}, \tilde{\ell}} \sim 20 \pm 10 \mathrm{TeV}$.

From these results, one may conclude that the present LHC Run 2 results of finding a SMlike Higgs boson with $m_{h}=125 \mathrm{GeV}$ and no sign of sparticles is seeing exactly that which the landscape with $n=1$ or 2 predicts will be seen. Furthermore, the higgsino-like WIMPs would form only a portion of dark matter (along with a SUSY DFSZ-like axion). Since the higgsino-like WIMPs typically constitute only $\sim 10 \%$ of dark matter, their detection rates lie below present WIMP search limits.

We also have checked the case with positive $A$ term. Sparticles are pulled to higher masses due to the statistical draw but the Higgs mass peaks at $120 \mathrm{GeV}$ and less than $1 \%$ of the scan points give correct Higgs mass. Since our main motivation for the landscape picture is the prediction of $m_{h}$ at its measured value, we do not consider experimental implications of $A_{0}>0$ for the remainder of the paper.

Our goal for the present paper is to confront the panoply of recent LHC and WIMP search experiment results with the predictions from the string theory landscape. 


\section{Landscape predictions vs. LHC search limits}

In this section, we confront the string theory landscape predictions for $n=1$ with LHC sparticle search constraints and projected reach limits.

\subsection{Landscape predictions for NUHM2 model}

In years past, it was common to portray collider search limits in the $m_{1 / 2}$ vs. $m_{0}$ plane of the mSUGRA/CMSSM model [31,32]. In this model, the matter and Higgs soft mass terms are unified to a common GUT scale value $m_{0}$, where the GUT scale is defined as that scale $m_{G U T} \simeq 2 \times 10^{16} \mathrm{GeV}$ where the gauge couplings $g_{1}$ and $g_{2}$ unify. In the mSUGRA/CMSSM model, since $m_{H_{u}}^{2}=m_{H_{d}}^{2} \equiv m_{0}^{2}$ as an input parameter, then $\mu$ is constrained by eq. (2.7) so as to ensure the measured value of $m_{Z}=91.2 \mathrm{GeV}$. The natural portion of parameter space using Barbieri-Giudice fine-tuning [33] was found to be the lowest allowed values of $m_{0}$ and $m_{1 / 2} \lesssim 200 \mathrm{GeV}$ [34]. This region is long-since excluded by LHC sparticle search constraints which with $80 \mathrm{fb}^{-1}$ of integrated luminosity now require $m_{\tilde{g}} \gtrsim 2.25 \mathrm{TeV}$ and $m_{\tilde{t}_{1}} \gtrsim 1.1 \mathrm{TeV}$.

The value of $\mu$ in the LHC-allowed region is only natural $\sim 100-300 \mathrm{GeV}$ in the focus point (FP) region [35-37]. But the FP region appears only for the smaller range of $A_{0}$ where $m_{h}$ is too low [38]. Thus, the region of mSUGRA/CMSSM parameter space with $m_{h} \sim 123-127 \mathrm{GeV}$ is always highly fine-tuned [39]. For this reason, we work instead first in the two-extra-parameter non-universal Higgs model NUHM2 [40-45] which allows independent input values of $m_{H_{u}}^{2}$ and $m_{H_{d}}^{2}$ (since the Higgs live in independent GUT multiplets anyway). The values of $m_{H_{u}}^{2}$ and $m_{H_{d}}^{2}$ may be traded for weak scale inputs $\mu$ and $m_{A}$. This allows us to adopt a natural value of $\mu \simeq 200 \mathrm{GeV}$ over all parameter space. We use Isajet 7.88 for our SUSY spectra generation and calculation of $\Delta_{\text {EW }}$ [46].

In figure 3 we display the $m_{1 / 2}$ vs. $m_{0}$ plane of the NUHM2 model for $\mu=200 \mathrm{GeV}$, $\tan \beta=10, A_{0}=-1.6 m_{0}$ and $m_{A}=2 \mathrm{TeV}$. The soft terms $m_{0}$ and $m_{1 / 2}$ are generated randomly for $m_{0}: 0 \rightarrow 10 \mathrm{TeV}$ and $m_{1 / 2}: 0.3-3 \mathrm{TeV}$ but with the $n=1$ increasing distribution and $\Delta_{\mathrm{EW}}<30$ so that the weak scale is within $\sim 4$ of its measured value. We see that the low $m_{0}$ and $m_{1 / 2}$ region is now sparsely populated due to the (mild) draw of the landscape towards large soft terms. In this plot, the density of points actually reflects the assumed vacuum statistics of the landscape with $n=1$. The density increases with increasing $m_{0}$ and $m_{1 / 2}$ until the points cut off where soft term contributions to the weak scale exceed the measured weak scale by a factor four $\left(\Delta_{\mathrm{EW}}>30\right)$. The red line denotes the latest LHC Run 2 bound of $m_{\tilde{g}} \gtrsim 2.25 \mathrm{TeV}$. The green points are LHC-allowed while black points above the red contour at lower $m_{0}$ have $m_{h}<123 \mathrm{GeV}$ (we assume an approximate $\pm 2 \mathrm{GeV}$ theory error in the Isajet $m_{h}$ calculation). The green LHC-allowed points range up to $m_{\tilde{g}} \sim 3.5 \mathrm{TeV}$ although for other parameter choices and moving to the NUHM3 model then gluinos can range as high as $6 \mathrm{TeV}$ [47]. The most densely populated region of parameter space remains beyond current LHC reach and it may require an upgrade to high energy LHC (HE-LHC with $\sqrt{s}=27 \mathrm{TeV}$ and $15 \mathrm{ab}^{-1}$ of integrated luminosity) to completely cover the remaining parameter space in the gluino pair production search channel [48]. 


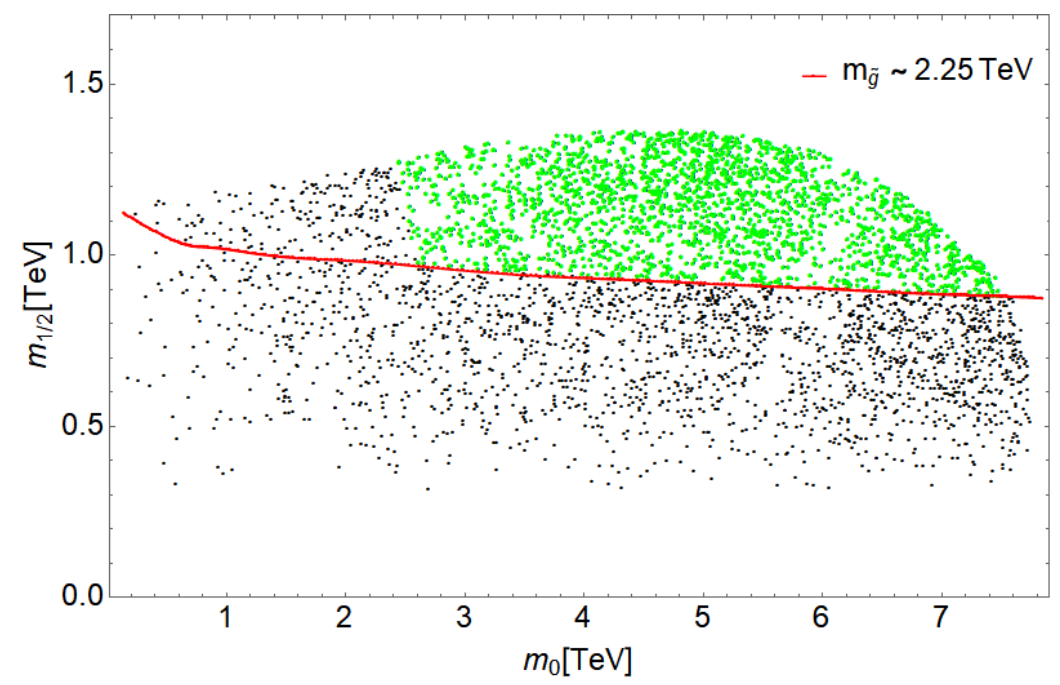

Figure 3. Locus of $n=1$ lansdscape scan points for the NUHM2 model with $\mu=200 \mathrm{GeV}$ in the $m_{1 / 2}$ vs. $m_{0}$ plane along with recent LHC Run2 constraints for $A_{0}=-1.6 m_{0}, m_{A}=2 \mathrm{TeV}$ and $\tan \beta=10$. The soft terms $m_{0}$ and $m_{1 / 2}$ are selected with $n=1$ and $\Delta_{\mathrm{EW}}<30$ so that the weak scale is within $\sim 4$ of its measured value. The red contour corresponds to the LHC Run 2 limit that $m_{\tilde{g}} \gtrsim 2.25 \mathrm{TeV}$. Green points are allowed by LHC Run 2 constraints (see text) while black points are excluded by LHC Run 2.

\subsection{Landscape predictions for NUHM3 model}

In this subsection, we investigate landscape predictions within the more general NUHM3 model where first/second generation matter scalars have different soft terms from third generation matter scalars. This sort of setup is motivated in part by investigations of the minilandscape of heterotic string models compactified on an orbifold [49]. In these models, the first/second generation multiplets live near orbifold fixed points and obey localized grand unification [50]: they live in the 16-dimensional spinor reps of $\mathrm{SO}(10)$. In contrast, the third generation matter scalars, Higgs multiplets and gauginos live more in the bulk of the compactified orbifold and hence live in the usual SM split multiplets.

In this section, we will scan over soft parameters as $m_{\text {soft }}^{n=1}$ with the following ranges:

- $m_{0}(1,2): 0-55 \mathrm{TeV}$,

- $m_{0}(3): 0-20 \mathrm{TeV}$,

- $m_{1 / 2}: 0-3.2 \mathrm{TeV}$,

- $-A_{0}: 0-25 \mathrm{TeV}$ and

- $m_{A}: 0-10 \mathrm{TeV}$,

while we scan over $\tan \beta: 3-60$ and $\mu: 100-360 \mathrm{GeV}$ uniformly since $\tan \beta$ is not dimensional and $\mu$ arises from our assumed solution to the SUSY $\mu$ problem. The lower limit of the $\mu$ term comes from the LEP2 limit on the lightest chargino mass, $\widetilde{\chi}_{1}^{ \pm}>103.5 \mathrm{GeV}$. 


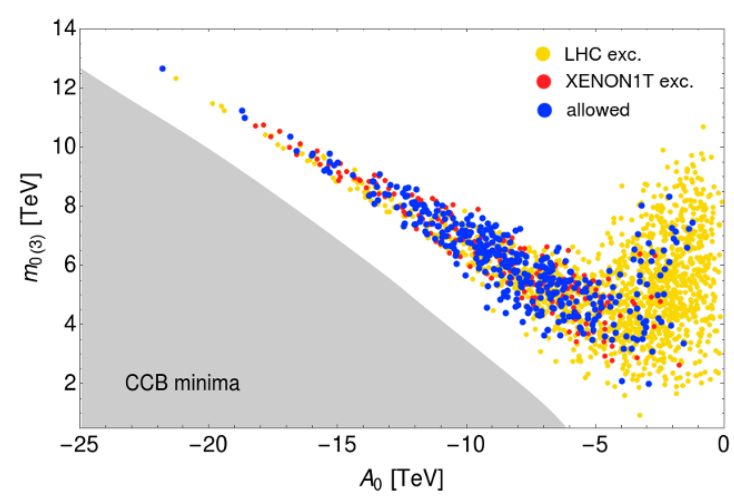

(a) We show regions of $\Delta_{\mathrm{EW}}>30$ (blank) and CCB minima (lower left) in the scalar potential. The blue points are LHC Run 2 and DMallowed.

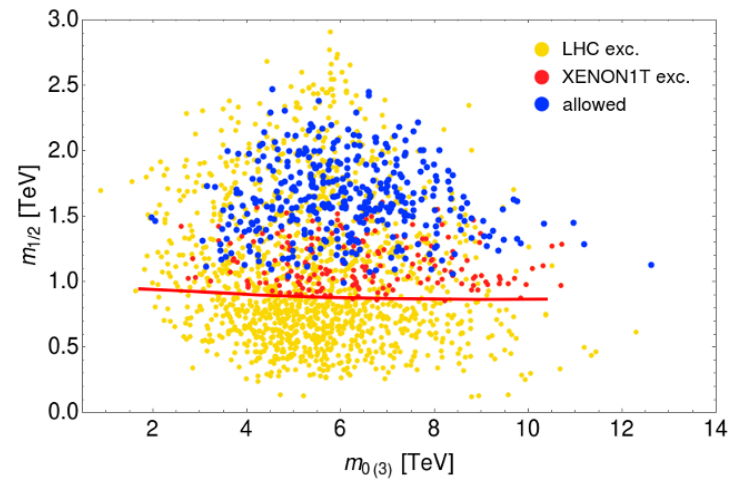

(b) LHC Run 2 limit for $m_{\tilde{g}}>2.25 \mathrm{TeV}$ is shown by the red contour.

Figure 4. Locus of $n=1$ landscape scan points in the (a) $m_{0}(3)$ vs. $A_{0}$ and (b) $m_{1 / 2}$ vs. $m_{0}(3)$ planes for the NUHM3 model with $\mu=100-360 \mathrm{GeV}$.

We again require an appropriate EWSB and further require no contributions of eq. (2.7) to the weak scale to exceed a factor four (i.e. $\Delta_{\mathrm{EW}}<30$ ).

Our first results are shown in figure 4 a where we show scan points in the $m_{0}(3)$ vs. $A_{0}$ plane. Here we divide our scan points into three sets. Yellow points are excluded by recent LHC Run 2 search limits:

- $m_{\tilde{g}} \gtrsim 2.25 \mathrm{TeV}$ for $\tilde{g} \rightarrow t \bar{t}+\widetilde{\chi}_{1}^{0}[51]$,

- $m_{\tilde{t}_{1}} \gtrsim 1.1 \mathrm{TeV}$ for $\tilde{t} \rightarrow t^{(*)}+\tilde{\chi}_{1}^{0}[52]$,

- bounds from $H / A \rightarrow \tau^{+} \tau^{-}$in the $\tan \beta$ vs. $m_{A}$ plane [53],

- higgsino pair production [54]: points are beyond the recent LHC soft dilepton + jets $+E_{T}$ constraints (shown later in figure $8 \mathrm{~b}$ ),

- $m_{h}=125 \pm 2 \mathrm{GeV}$ (to account for theory error of the calculation)

The blue shaded points have acceptable vacua and obey both LHC and WIMP search constraints. The red points are LHC-allowed but excluded by recent XENON1T spinindependent (SI) direct WIMP detection (DD) searches [55] (see later figure 10). The main lesson from figure $4 \mathrm{a}$ is that the acceptable points lie in a very restricted regions where $m_{0}(3)$ and $-A_{0}$ are correlated: if $A_{0}$ gets too large (negative), then the model is forced into CCB minima so the gray region is disallowed. Likewise, if for fixed $A_{0}$ then $m_{0}(3)$ gets too large, then third generation contributions to the weak scale $\Sigma_{u}^{u}\left(\tilde{t}_{1,2}\right)$ exceed the measured weak scale by over a factor four (blank region).

In figure $4 \mathrm{~b}$ we show the $m_{1 / 2}$ vs. $m_{0}(3)$ soft term plane. The LHC Run 2 requirement that $m_{\tilde{g}} \gtrsim 2.25 \mathrm{TeV}$ is shown by the red contour. There are plenty of surviving landscape scan points with $m_{1 / 2}$ ranging from $1-2.5 \mathrm{TeV}$. The upper range of allowed $m_{1 / 2}$ values 


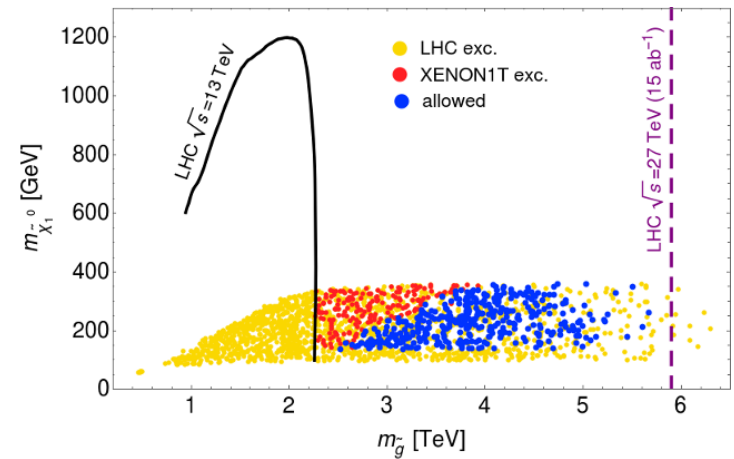

(a)

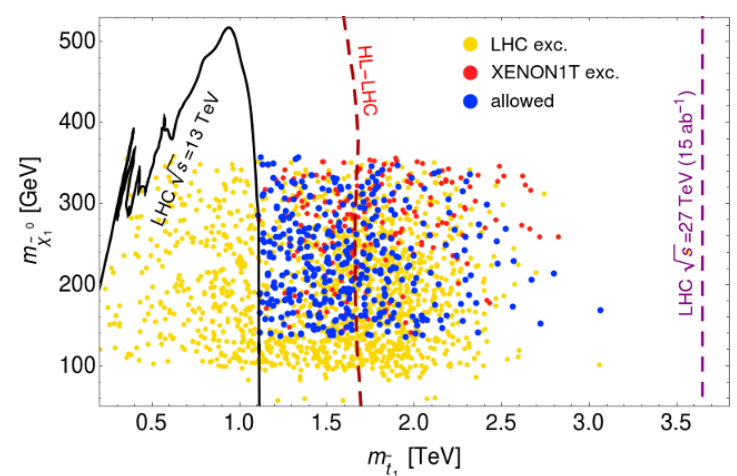

(b)

Figure 5. Locus of $n=1$ landscape scan points for the NUHM3 model with $\mu=100-360 \mathrm{GeV}$ in the (a) $m_{\tilde{\chi}_{1}^{0}}$ vs. $m_{\tilde{g}}$ and (b) $m_{\tilde{\chi}_{1}^{0}}$ vs. $m_{\tilde{t}_{1}}$ planes versus recent LHC Run2 constraints.

correspond to values of $m_{\tilde{g}}$ over $6 \mathrm{TeV}$. Few scan points exist for $m_{0}(3) \lesssim 2 \mathrm{TeV}$ since the $n=1$ scan prefers linearly increasing soft terms. Few points also exist for $m_{0}(3) \gtrsim 12 \mathrm{TeV}$ since these points would give too large $\Sigma_{u}^{u}\left(\tilde{t}_{1,2}\right)$ contributions to the weak scale.

In figure 5 a, we show our $n=1$ landscape scan points in the $m_{\widetilde{\chi}_{1}^{0}}$ vs. $m_{\tilde{g}}$ plane which is the usual simplified model plane in which LHC gluino searches are usually presented. The current LHC Run 2 exclusion contour based on $80 \mathrm{fb}^{-1}$ of integrated luminosity is shown as the black contour. It is also interesting that the XENON1T dark matter search excludes significant regions of the lighter LSP masses for gluino masses of order 2-3.5 TeV. HL-LHC will be able to cover points with gluinos only up to $2.8 \mathrm{TeV}$ via the gluino pair production channel [56]. We also show the recently computed 95\% CL HE-LHC projected search limit for $\sqrt{s}=27 \mathrm{TeV}$ and $15 \mathrm{ab}^{-1}$ which reaches to $m_{\tilde{g}} \sim 6 \mathrm{TeV}$. Evidently a complete examination of $n=1$ landscape points in the $\tilde{g} \tilde{g}$ search channels will require HE-LHC.

In figure $5 \mathrm{~b}$, we show $n=1$ landscape points in the $m_{\widetilde{\chi}_{1}^{0}}$ vs. $m_{\tilde{t}_{1}}$ simplified model plane. The current LHC Run 2 search limits are shown as the black contour. There is a high density of LHC-allowed (blue) points extending from $m_{\tilde{t}_{1}} \sim 1.1$ to $2.7 \mathrm{TeV}$. The projected reach of HL-LHC with $\sqrt{s}=14 \mathrm{TeV}$ and $3 \mathrm{ab}^{-1}$ extends to $m_{\tilde{t}_{1}} \sim 1.7 \mathrm{TeV}$ and covers perhaps the greatest density of blue points. Nonetheless, it will require an upgrade to HE-LHC to cover the complete set of $n=1$ landscape points [48].

In figure 6 , we show the $n=1$ landscape points in the $m_{\tilde{g}}$ vs. $m_{\tilde{t}_{1}}$ plane. Of note here is that the points with the largest values of $m_{\tilde{g}}$ have the smaller range of $m_{\tilde{t}_{1}}$ and vice-versa. Thus, if somehow for instance gluinos were able to escape LHC detection on account of them being too heavy, the top squarks would surely be seen (and vice-versa). A complete coverage of the $n=1$ landscape parameter space will require HE-LHC with 15 $a b^{-1}$ of integrated luminosity.

In figure 7 , we show the $m_{0}(1,2)$ vs. $m_{0}(3)$ plane of the NUHM3 model for the $n=1$ landscape. The important lesson from this plot is that first/second generation matter scalar soft terms tend to inhabit the $10-30 \mathrm{TeV}$ range whilst third generation matter scalar soft terms lie typically below $10 \mathrm{TeV}$. RG and mixing effects then cause the third generation 


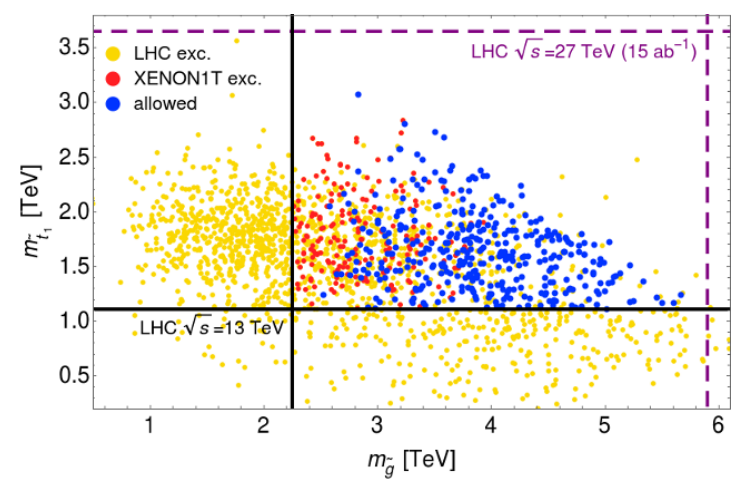

Figure 6. Locus of $n=1$ landscape scan points for the NUHM3 model with $\mu=100-360 \mathrm{GeV}$ in the $m_{\tilde{t}_{1}}$ vs. $m_{\tilde{g}}$ plane versus recent LHC Run2 constraints (black) and projected HE-LHC 95\% CL reach contours (purple-dashed).

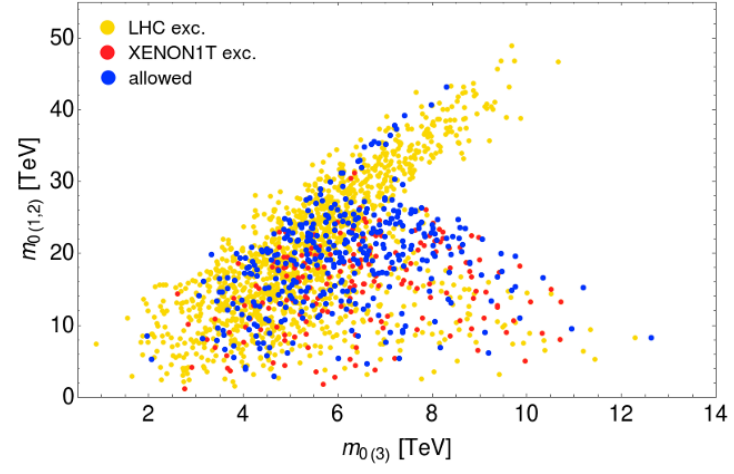

Figure 7. Locus of $n=1$ landscape scan points for the NUHM3 model in the $m_{0}(1,2)$ vs. $m_{0}(3)$ plane for $\mu=100-360 \mathrm{GeV}$.

squarks/sleptons to lie in the few $\mathrm{TeV}$ range (so their loop-suppressed contributions $\Sigma_{u}^{u}$ to the weak scale are small) while first/second generation squarks and slepton (with mass $\left.m_{\tilde{q}, \tilde{\ell}} \sim m_{0}(1,2)\right)$ lie well beyond even HE-LHC reach and offer at least a partial decoupling solution to the SUSY flavor and CP problems. The reason they can be so heavy is that the first/second generation sfermion contributions to the weak scale are $D$-term contributions which largely cancel [57].

Another important LHC search plane is the $\mu$ vs. $m_{\widetilde{\chi}_{2}^{ \pm}}$plane This plane is important for presenting search limits from same-sign diboson (SSdB) production arising from wino pair production in SUSY models with light higgsinos. The reaction is $p p \rightarrow \widetilde{\chi}_{2}^{ \pm} \widetilde{\chi}_{4}^{0}$ where $\tilde{\chi}_{2}^{ \pm} \rightarrow W^{ \pm} \widetilde{\chi}_{1,2}^{0}$ while $\tilde{\chi}_{4}^{0} \rightarrow W^{\mp} \tilde{\chi}_{1}^{ \pm}$so that half the time one arrives at a final state with two same-sign $W$ bosons plus large $\mathbb{E}_{T}$. For leptonically-decaying $W$ bosons, then the final state consists of a same-sign dilepton $+E_{T}$ signature which is relatively jet free- in contrast to same-sign dileptons originating from gluino and squark pair production. The same-sign channel has rather tiny SM backgrounds [58-60]. So far, no search results have been presented by ATLAS or CMS. From figure 8a, we see that LHC-allowed points only begin at wino masses $m_{\widetilde{\chi}_{2}^{ \pm}} \sim 800 \mathrm{GeV}$ and then extend out to $m_{\widetilde{\chi}_{2}^{ \pm}} \sim 2300 \mathrm{GeV}$. This is to be compared with the projected HL-LHC 95\% CL search limit which is the brown contour reaching to $m_{\widetilde{\chi}_{2}^{ \pm}} \sim 1100 \mathrm{GeV}$ [60]. Projected search limits for HE-LHC in the SSdB channel have yet to be computed.

In figure $8 \mathrm{~b}$, we show the $n=1$ landscape points in the $\Delta m \equiv m_{\widetilde{\chi}_{2}^{0}}-m_{\widetilde{\chi}_{1}^{0}}$ vs. $m_{\widetilde{\chi}_{2}^{0}}$ plane. This plane is important for the light higgsino pair production searches $p p \rightarrow \widetilde{\chi}_{2}^{0} \widetilde{\chi}_{1}^{0}$ (and $\left.\tilde{\chi}_{2}^{0} \widetilde{\chi}_{1}^{ \pm}\right)$where $\tilde{\chi}_{2}^{0} \rightarrow \ell^{+} \ell^{-} \widetilde{\chi}_{1}^{0}$ giving rise to a soft opposite-sign dilepton pair whose invariant mass is bounded by $m_{\widetilde{\chi}_{2}^{0}}-m_{\widetilde{\chi}_{1}^{0}}$ [61-63]. To trigger on such events, it seems necessary to require a hard jet radiation from the initial state against which the soft dileptons can recoil. Recent search limits have been presented by both ATLAS [54] and CMS [64]. We show as a black contour the recent ATLAS limit. An important point of the $n=1$ landscape is that it favors heavier gauginos while $\mu$ must not be too far from the weak scale. The 


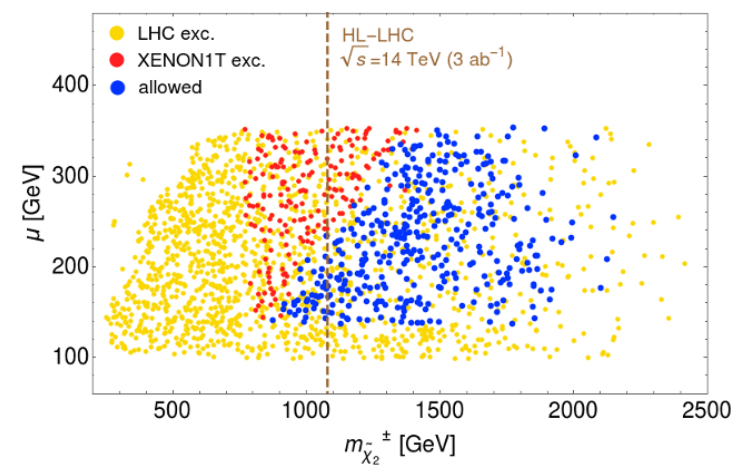

(a)

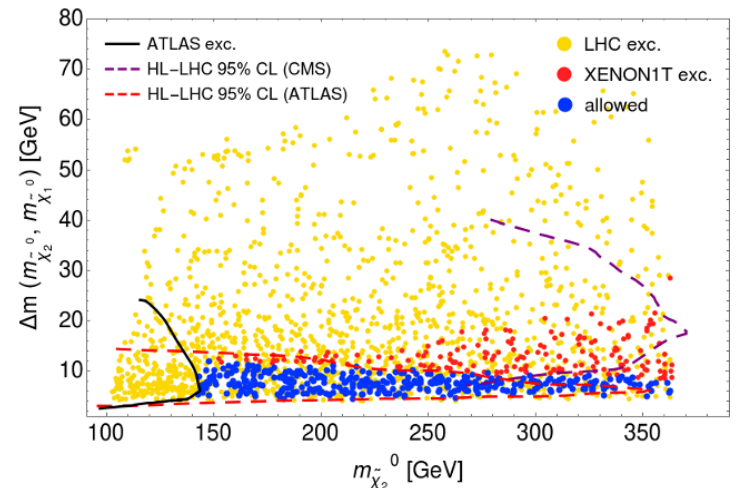

(b)

Figure 8. Locus of $n=1$ landscape scan points for the NUHM3 model with $\mu=100-360 \mathrm{GeV}$ in the (a) $\mu$ vs. $m_{\widetilde{\chi}_{2}^{ \pm}}$and (b) $m_{\widetilde{\chi}_{2}^{0}}-m_{\widetilde{\chi}_{1}^{0}}$ vs. $m_{\widetilde{\chi}_{2}^{0}}$ planes versus projected HL-LHC $95 \%$ CL search limits.

combination squeezes down the inter-higgsino mass gap $m_{\widetilde{\chi}_{2}^{0}}-m_{\widetilde{\chi}_{1}^{0}}$ so that in this case all the LHC-allowed points have $\Delta m \lesssim 10 \mathrm{GeV}$. We also show recently computed projected HL-LHC 95\% CL reach contours [65]. The ATLAS contour has focussed on the small mass gap region and appears to cover nearly all parameter space. This has important implications for how SUSY is likely to be revealed at LHC. Gluinos and top squarks are expected to be drawn to large values, possibly beyond HL-LHC reach. The soft dilepton plus jet $+E_{T}$ channel (SDLJMET) is the only channel that seems to be (nearly) completely covered by HL-LHC. Thus, we would expect a SUSY signal in this channel to emerge slowly but conclusively during the next 15 years as LHC acquires its full complement of 3 $a b^{-1}$ of integrated luminosity!

A final natural SUSY search channel occurs in the Higgs sector by looking for $p p \rightarrow$ $A, H \rightarrow \tau^{+} \tau^{-}$events. These searches are typically presented in the $\tan \beta$ vs. $m_{A}$ plane which we show in figure 9 . We also show the recent LHC excluded region as the black contour [53]. This latter contour assumes only SM decay modes for the $A$ and $H$ but for the landscape then the decay modes $H, A \rightarrow$ higgsinos should almost always be open as well (and might lead to $4 \ell+E_{T}$ signatures [66]). The added $H, A \rightarrow$ higgsinos decay modes hardly affect the search limits since a diminution of Higgs to SM branching fractions can be offset by increasing the Higgs production cross sections by moving to somewhat larger $\tan \beta$ [67]. From the plot, we see that the LHC-allowed points are typically well beyond the current LHC reach limits and the greatest density populates the region of $m_{A} \sim 2-5 \mathrm{TeV}$ and at lower $\tan \beta$ values where $b \bar{b}$ fusion contributions to the production cross section are not so big. Thus, we see only a small likelihood of a signal emerging in this channel at LHC.

\section{Landscape predictions vs. WIMP DM search limits}

In $n=1$ landscape SUSY, we expect soft terms to be drawn to large values whilst the $\mu$ term is not too far from the weak scale. This results in a Little Hierarchy (LH) with $\mu \ll m_{\text {soft }}$ which turns out to be non-problematic. In such a scenario, then the lightest 


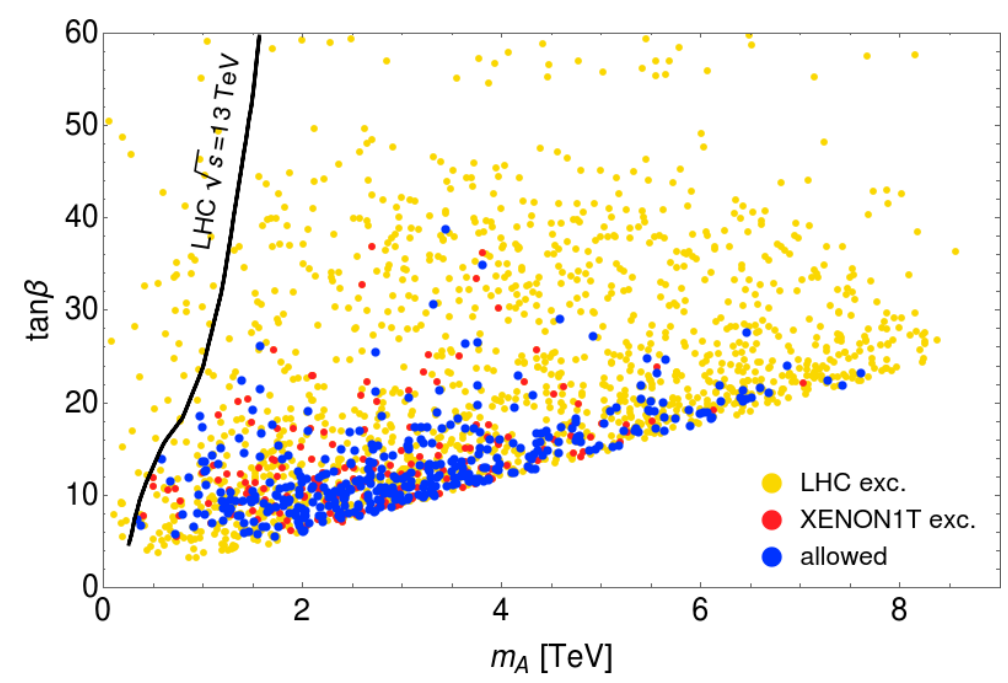

Figure 9. Locus of $n=1$ landscape scan points for the NUHM3 model with $\mu=100-360 \mathrm{GeV}$ in the $\tan \beta$ vs. $m_{A}$ plane versus recent LHC Run2 constraints. Blue points are LHC Run 2 and DM-allowed while red points are LHC-safe but excluded by XENON1T WIMP search limits.

SUSY particle is the lightest higgsino-like neutralino with mass $m_{\widetilde{\chi}_{1}^{0}} \sim 90-360 \mathrm{GeV}$. These natural higgsino-like WIMPs are thermally underproduced as dark matter, which may be a reason why they had not been considered much previous to 2011 [68-70]. However, one must recall that the QCD sector of the SM also suffers a naturalness issue in the form of the strong CP problem. Including an axion sector into the MSSM is thus well-motivated both for solving the strong CP problem but also to solve the SUSY $\mu$ problem (for a recent review, see e.g. ref. [71]) via a DFSZ-type SUSY axion. Thus, in natural SUSY it is expected that the DM is a WIMP-axion admixture (i.e. two dark matter particles). If the natural SUSY WIMPs (with $m_{\tilde{\chi}_{1}^{0}} \lesssim 350 \mathrm{GeV}$ ) were all of DM, then they would actually be excluded by current WIMP search constraints [72]. But if the DM is mainly axions, then there are far fewer relic WIMPs present in the cosmos and they can escape present limits from WIMP search experiments. A full evaluation of mixed WIMP-axion dark matter requires an eight-coupled Boltzmann equation evaluation which accounts also for axino, saxion and gravitino production and decay in the early universe [73].

We first examine WIMP search limits via ton-scale noble liquid experiments using targets such as Xenon or Argon. To compare WIMP search limits to landscape projections, one must calculate $\xi \sigma^{S I}\left(\widetilde{\chi}_{1}^{0}, p\right)$ where $\xi \equiv \Omega_{\widetilde{\chi}_{1}^{0}} h^{2} / 0.12$, i.e. it is the fractional abundance of WIMPs in making up the totality of dark matter. Usually this is just the WIMP thermal abundance divided by the measured abundance although it is possible the WIMP abundance is supplemented by non-thermal processes such as axino or saxion decay in the early universe.

In figure 10 , we plot the locus of $n=1$ landscape points in the $\xi \sigma^{S I}\left(\widetilde{\chi}_{1}^{0}, p\right)$ vs. $m_{\widetilde{\chi}_{1}^{0}}$ plane. We also show current search limits from the XENON-100 experiment [74] (black contour) and the XENON1T experiment [55] (red contour). A subset of LHC-allowed points are already excluded, and denoted as red points. However, the bulk of $n=1$ 


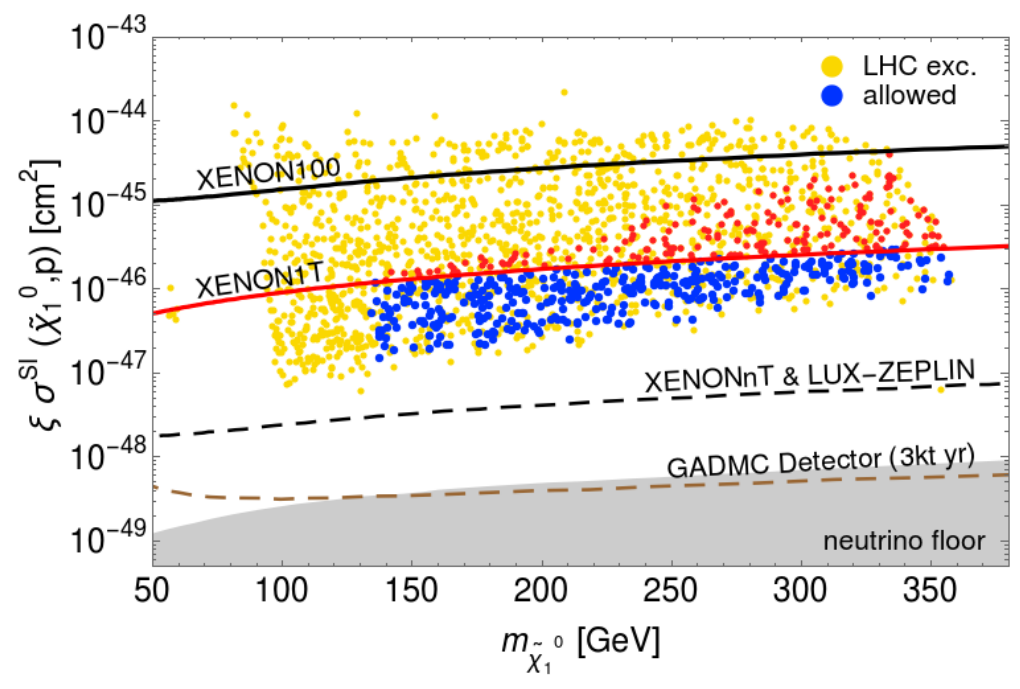

Figure 10. Locus of $n=1$ landscape scan points for the NUHM3 model in the $\xi \sigma^{S I}\left(\widetilde{\chi}_{1}^{0}, p\right)$ vs. $m_{\widetilde{\chi}_{1}^{0}}$ plane versus recent WIMP search constraints for $\mu=100-360 \mathrm{GeV}$. Red points are excluded by XENON1T search limits but not by LHC Run 2 constraints. Projected reaches from several future SI DD experimentes are also shown.

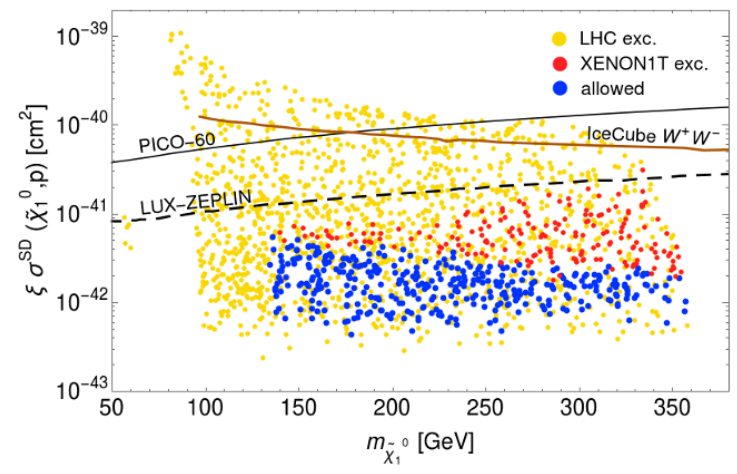

(a) Spin-dependent DM detection rates

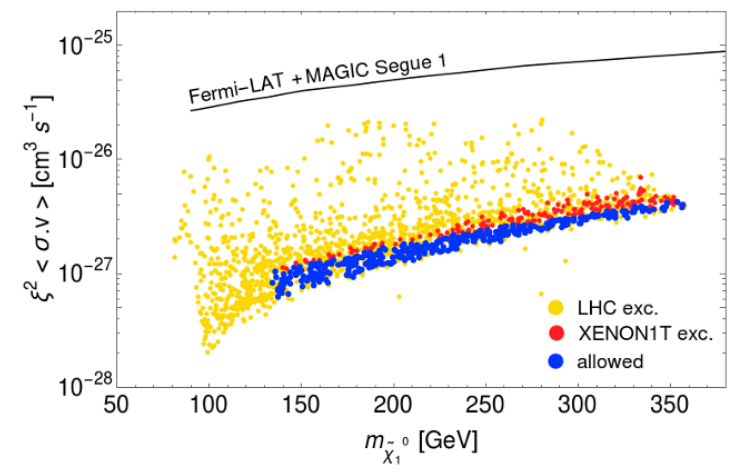

(b) Indirect DM detection rates

Figure 11. Locus of $n=1$ landscape scan points for the NUHM3 model with $\mu=100-360 \mathrm{GeV}$ in the (a) $\xi \sigma^{S D}\left(\widetilde{\chi}_{1}^{0}, p\right)$ vs. $m_{\widetilde{\chi}_{1}^{0}}$ and (b) $\xi^{2}\langle\sigma v\rangle$ vs. $m_{\widetilde{\chi}_{1}^{0}}$ planes versus recent WIMP search constraints.

landscape points are still allowed, and extend down to an order of magnitude below present limits. These points do not extend all the way to the neutrino floor since in SUSY the WIMPs couple to nucleons mainly via light Higgs exchange and this coupling involves a production of gaugino times higgsino components [70]. In natural SUSY, the WIMP is mainly higgsino, but with non-negligible gaugino component (lest heavy gauginos give too large a contribution to the weak scale). Thus, it appears that projected seach limits from XENONnT (multi-ton Xenon detector), LUX-ZEPLIN (LZ) [75] and other multi-ton-scale detectors $[76,77]$ should cover the entire $n=1$ landscape parameter space, even if WIMPs comprise only a portion of the dark matter.

In figure 11a, we show the spin-dependent (SD) direct detection scattering rate $\xi \sigma^{S D}\left(\widetilde{\chi}_{1}^{0}, p\right)$ vs. $m_{\widetilde{\chi}_{1}^{0}}$ plane along with projected $n=1$ landscape rates. We also show 
recent limits from the PICO-60 experiment [78] and IceCube in the $W^{+} W^{-}$annihilation mode [79]. In this case, the LHC-allowed landscape points tend to lie about an order of magnitude below the current limits. We also show the projected future reach of LZ [75]. Even this contour does not quite reach the expected theory region.

In figure $11 \mathrm{~b}$, we show the indirect WIMP detection rates (IDD) in the $\xi^{2}\langle\sigma \cdot v\rangle$ vs. $m_{\widetilde{\chi}_{1}^{0}}$ plane, where $\xi^{2}$ is required since these signals arise from WIMP-WIMP annihilation in the cosmos and is thus suppressed by the fractional WIMP abundance squared. We show also the recent Fermi-LAT+Magic limits from observation of dwarf spheroidal galaxy Segue1 [80]. The current limit is over an order of magnitude above the expected LHC-allowed points from the $n=1$ landscape. A signal from the IDD search channel would point to non-thermal production of WIMPs (i.e. from decays of axinos/saxions) in addition to the thermally-produced neutralinos.

\section{Conclusions}

Rather general arguments regarding the statistics of the landscape of flux vacua in string theory point to a statistical draw towards large soft SUSY breaking terms governed by a power law $m_{\text {soft }}^{n}$ where $n=2 n_{F}+n_{D}-1$ involves the number of $F$ and $D$ term SUSY breaking fields in a (possibly) complicated hidden sector. With only the draw towards large soft terms, one would expect a huge value for the weak scale since $m_{\text {weak }}$ is determined by the visible sector soft terms and the SUSY $\mu$ parameter. A huge weak scale would mean highly suppressed weak interactions and huge particle masses which would likely lead to a non life-supporting universe. Agrawal et al. calculated that an increase in $m_{\text {weak }}$ by a factor of $\sim 3$ would lead to a non-livable universe. Therefore, we have tempered the statistical draw of the landscape to large soft terms with the anthropic requirement of a weak scale no more than (conservatively) four times its measured value. For a fixed natural value of $\mu$ (arising from some solution to the SUSY $\mu$ problem such as hybrid CCK which also solves the strong CP problem and introduces axionic along with higgsino-like WIMP dark matter [30]), then one may implement random scans over power-law increasing soft terms tempered by an anthropic requirement of the weak scale not too far from $m_{\text {weak }} \sim 100 \mathrm{GeV}$. The cases for $n=1$ and 2 lead to a landscape probability distribution for the light Higgs mass which peaks around $\sim 125 \mathrm{GeV}$. Also, most superparticle masses are pulled to large values beyond LHC reach. In this case, LHC sparticle and WIMP dark matter search experiments are seeing exactly that which is expected from the $n=1,2$ landscape: a Higgs mass of $125 \mathrm{GeV}$ and no sign yet of sparticles.

Our goal in this paper was a practical one: place the $n=1$ landscape statistical predictions on the same plots that LHC sparticle and WIMP dark matter search experiments use in order to assess where sparticle and WIMP masses might be located relative to present and future search limits. In these sorts of plots, the density of points actually has meaning since it would reflect the assumed statistics of string theory vacua in a fertile patch which includes the MSSM as the weak scale effective theory.

Our findings are that strongly interacting sparticles $\tilde{g}$ and $\tilde{t}_{1}$ are likely to lie beyond present LHC search bounds and possibly beyond HL-LHC projected search limits. It may 
require an upgrade to HE-LHC to cover the entire $n=1$ landscape parameter space in the $\tilde{g} \tilde{g}$ and $\tilde{t}_{1} \tilde{t}_{1}^{*}$ modes as manifested in the context of the natural NUHM3 SUSY model. Also, the SSdB signal may or may not be detected by HL-LHC and the $A, H \rightarrow \tau^{+} \tau^{-}$signals are likely to lie well beyond the reach of LHC upgrades. However, the SDLJMET signal arising from higgsino pair production offers a search channel wherein HL-LHC may cover the entire projected parameter space. In this case, we would expect a SUSY signal to emerge slowly but conclusively by ATLAS and CMS as more and more integrated luminosity accrues.

Regarding WIMP searches, it appears that a full complement of data from multiton noble liquid SI direct detection experiments should cover the entire $n=1$, NUHM3 landscape parameter space. This can occur even though in natural SUSY the higgsino-like WIMPs make up only a portion of dark matter with SUSY DFSZ axions making up the remainder. Meanwhile, even upgraded SD detectors may well fall short of covering the portion of parameter space occupied by the $n=1$ landscape model. It appears that IDD WIMP search experiments will also have a hard time accessing the full parameter space since now the expected signal rates are diminished by the square of the fractional WIMP abundance. The search for SUSY DFSZ axions, addressed in ref. [81], is also difficult due to the presence of higgsinos circulating in the $a \gamma \gamma$ coupling diagram: they tend to suppress the DFSZ axion coupling to much lower values than are typically displayed in axion search result plots.

To summarize:

- The $n=1$ landscape statistics predict $m_{h} \sim 125 \mathrm{GeV}$ with superparticles beyond the reach of present LHC and WIMP DD experiments.

- LHC signals in SDLJMET channel should emerge slowly as LHC attains higher and higher integrated luminosity. A signal should likely be seen with $3 \mathrm{ab}^{-1}$ at HL-LHC if not sooner. Signals in other channels such as $\tilde{g} \tilde{g}, \tilde{t}_{1} \tilde{t}_{1}^{*}$ and SSdB may emerge at HL-LHC if we are lucky but otherwise may require an upgrade to HE-LHC.

- WIMP detection signals should emerge in SI DD experiments using multi-ton noble liquids. Signals in SD DD or IDD are much less likely to emerge in the near future.

\section{Acknowledgments}

We thank Art McDonald for discussions on future WIMP detectors. This work was supported in part by the US Department of Energy, Office of High Energy Physics. The computing for this project was performed at the OU Supercomputing Center for Education \& Research (OSCER) at the University of Oklahoma (OU).

Open Access. This article is distributed under the terms of the Creative Commons Attribution License (CC-BY 4.0), which permits any use, distribution and reproduction in any medium, provided the original author(s) and source are credited. 


\section{References}

[1] R. Bousso and J. Polchinski, Quantization of four form fluxes and dynamical neutralization of the cosmological constant, JHEP 06 (2000) 006 [hep-th/0004134] [INSPIRE].

[2] L. Susskind, The Anthropic landscape of string theory, hep-th/0302219 [INSPIRE].

[3] R. Bousso and J. Polchinski, The string theory landscape, Sci. Am. 291 (2004) 60.

[4] S. Weinberg, Anthropic Bound on the Cosmological Constant, Phys. Rev. Lett. 59 (1987) 2607 [INSPIRE].

[5] S. Weinberg, The Cosmological Constant Problem, Rev. Mod. Phys. 61 (1989) 1 [inSPIRE].

[6] M.R. Douglas, The Statistics of string/M theory vacua, JHEP 05 (2003) 046 [hep-th/0303194] [INSPIRE].

[7] S. Ashok and M.R. Douglas, Counting flux vacua, JHEP 01 (2004) 060 [hep-th/0307049] [INSPIRE].

[8] S. Weinberg, Living in the multiverse, in Universe or multiverse?, B. Carr ed., pp. 29-42 (2005), hep-th/0511037 [INSPIRE].

[9] V. Agrawal, S.M. Barr, J.F. Donoghue and D. Seckel, Viable range of the mass scale of the standard model, Phys. Rev. D 57 (1998) 5480 [hep-ph/9707380] [INSPIRE].

[10] V. Agrawal, S.M. Barr, J.F. Donoghue and D. Seckel, Anthropic considerations in multiple domain theories and the scale of electroweak symmetry breaking, Phys. Rev. Lett. 80 (1998) 1822 [hep-ph/9801253] [INSPIRE].

[11] M.R. Douglas, Basic results in vacuum statistics, Comptes Rendus Physique 5 (2004) 965 [hep-th/0409207] [INSPIRE].

[12] L. Susskind, Supersymmetry breaking in the anthropic landscape, hep-th/0405189 [INSPIRE].

[13] F. Denef and M.R. Douglas, Distributions of flux vacua, JHEP 05 (2004) 072 [hep-th/0404116] [INSPIRE].

[14] M.R. Douglas, Statistical analysis of the supersymmetry breaking scale, hep-th/0405279 [INSPIRE].

[15] M.R. Douglas, The string landscape and low-energy supersymmetry, Les Houches Lect. Notes 97 (2015) 315.

[16] M. Dine, E. Gorbatov and S.D. Thomas, Low energy supersymmetry from the landscape, JHEP 08 (2008) 098 [hep-th/0407043] [INSPIRE].

[17] M. Dine, Supersymmetry, naturalness and the landscape, in Themes in Unification. Proceedings: 10th International Symposium on particles, strings and cosmology, (PASCOS 2004), Pran Nath Festschrift, Part II: Boston, U.S.A., August 18-19, 2004, pp. 249-263 (2004) [DOI:10.1142/9789812701756_0093] [hep-th/0410201] [INSPIRE].

[18] F. Denef, M.R. Douglas and S. Kachru, Physics of String Flux Compactifications, Ann. Rev. Nucl. Part. Sci. 57 (2007) 119 [hep-th/0701050] [inSPIRE].

[19] J. Kumar, A Review of distributions on the string landscape, Int. J. Mod. Phys. A 21 (2006) 3441 [hep-th/0601053] [INSPIRE].

[20] H.P. Nilles, Supersymmetry, Supergravity and Particle Physics, Phys. Rept. 110 (1984) 1 [INSPIRE]. 
[21] N. Arkani-Hamed and S. Dimopoulos, Supersymmetric unification without low energy supersymmetry and signatures for fine-tuning at the LHC, JHEP 06 (2005) 073 [hep-th/0405159] [INSPIRE].

[22] H. Baer, V. Barger, H. Serce and K. Sinha, Higgs and superparticle mass predictions from the landscape, JHEP 03 (2018) 002 [arXiv:1712.01399] [INSPIRE].

[23] J.A. Casas, A. Lleyda and C. Muñoz, Strong constraints on the parameter space of the MSSM from charge and color breaking minima, Nucl. Phys. B 471 (1996) 3 [hep-ph/9507294] [INSPIRE].

[24] H. Baer, M. Brhlik and D. Castano, Constraints on the minimal supergravity model from nonstandard vacua, Phys. Rev. D 54 (1996) 6944 [hep-ph/9607465] [INSPIRE].

[25] G.F. Giudice and R. Rattazzi, Living Dangerously with Low-Energy Supersymmetry, Nucl. Phys. B 757 (2006) 19 [hep-ph/0606105] [INSPIRE].

[26] H. Baer, V. Barger, M. Savoy and H. Serce, The Higgs mass and natural supersymmetric spectrum from the landscape, Phys. Lett. B 758 (2016) 113 [arXiv:1602.07697] [INSPIRE].

[27] H. Baer, V. Barger, P. Huang, A. Mustafayev and X. Tata, Radiative natural SUSY with a 125 GeV Higgs boson, Phys. Rev. Lett. 109 (2012) 161802 [arXiv:1207.3343] [INSPIRE].

[28] H. Baer, V. Barger, P. Huang, D. Mickelson, A. Mustafayev and X. Tata, Radiative natural supersymmetry: Reconciling electroweak fine-tuning and the Higgs boson mass, Phys. Rev. D 87 (2013) 115028 [arXiv:1212.2655] [INSPIRE].

[29] H. Baer and X. Tata, Weak scale supersymmetry: From superfields to scattering events, Cambridge University Press, Cambridge, U.K. (2006) [INSPIRE].

[30] H. Baer, V. Barger and D. Sengupta, Gravity safe, electroweak natural axionic solution to strong CP and SUSY $\mu$ problems, Phys. Lett. B 790 (2019) 58 [arXiv:1810.03713] [INSPIRE].

[31] G.L. Kane, C.F. Kolda, L. Roszkowski and J.D. Wells, Study of constrained minimal supersymmetry, Phys. Rev. D 49 (1994) 6173 [hep-ph/9312272] [INSPIRE].

[32] H. Baer, C.-H. Chen, R.B. Munroe, F.E. Paige and X. Tata, Multichannel search for minimal supergravity at $p \bar{p}$ and $e^{+} e^{-}$colliders, Phys. Rev. D 51 (1995) 1046 [hep-ph/9408265] [INSPIRE].

[33] R. Barbieri and G.F. Giudice, Upper Bounds on Supersymmetric Particle Masses, Nucl. Phys. B 306 (1988) 63 [INSPIRE].

[34] G.W. Anderson and D.J. Castano, Challenging weak scale supersymmetry at colliders, Phys. Rev. D 53 (1996) 2403 [hep-ph/9509212] [INSPIRE].

[35] K.L. Chan, U. Chattopadhyay and P. Nath, Naturalness, weak scale supersymmetry and the prospect for the observation of supersymmetry at the Tevatron and at the CERN LHC, Phys. Rev. D 58 (1998) 096004 [hep-ph/9710473] [INSPIRE].

[36] J.L. Feng, K.T. Matchev and T. Moroi, Multi-TeV scalars are natural in minimal supergravity, Phys. Rev. Lett. 84 (2000) 2322 [hep-ph/9908309] [INSPIRE].

[37] J.L. Feng, K.T. Matchev and T. Moroi, Focus points and naturalness in supersymmetry, Phys. Rev. D 61 (2000) 075005 [hep-ph/9909334] [INSPIRE].

[38] H. Baer, V. Barger and A. Mustafayev, Neutralino dark matter in mSUGRA/CMSSM with a 125 GeV light Higgs scalar, JHEP 05 (2012) 091 [arXiv:1202.4038] [INSPIRE]. 
[39] H. Baer, V. Barger, D. Mickelson and M. Padeffke-Kirkland, SUSY models under siege: LHC constraints and electroweak fine-tuning, Phys. Rev. D 89 (2014) 115019 [arXiv:1404.2277] [INSPIRE].

[40] D. Matalliotakis and H.P. Nilles, Implications of nonuniversality of soft terms in supersymmetric grand unified theories, Nucl. Phys. B 435 (1995) 115 [hep-ph/9407251] [INSPIRE].

[41] M. Olechowski and S. Pokorski, Electroweak symmetry breaking with nonuniversal scalar soft terms and large tan beta solutions, Phys. Lett. B 344 (1995) 201 [hep-ph/9407404] [INSPIRE].

[42] P. Nath and R.L. Arnowitt, Nonuniversal soft SUSY breaking and dark matter, Phys. Rev. D 56 (1997) 2820 [hep-ph/9701301] [INSPIRE].

[43] J.R. Ellis, K.A. Olive and Y. Santoso, The MSSM parameter space with nonuniversal Higgs masses, Phys. Lett. B 539 (2002) 107 [hep-ph/0204192] [INSPIRE].

[44] J.R. Ellis, T. Falk, K.A. Olive and Y. Santoso, Exploration of the MSSM with nonuniversal Higgs masses, Nucl. Phys. B 652 (2003) 259 [hep-ph/0210205] [INSPIRE].

[45] H. Baer, A. Mustafayev, S. Profumo, A. Belyaev and X. Tata, Direct, indirect and collider detection of neutralino dark matter in SUSY models with non-universal Higgs masses, JHEP 07 (2005) 065 [hep-ph/0504001] [INSPIRE].

[46] F.E. Paige, S.D. Protopopescu, H. Baer and X. Tata, ISAJET 7.69: A Monte Carlo event generator for $p p, \bar{p} p$ and $e^{+} e^{-}$reactions, hep-ph/0312045 [INSPIRE].

[47] H. Baer, V. Barger, J.S. Gainer, H. Serce and X. Tata, Reach of the high-energy LHC for gluinos and top squarks in SUSY models with light Higgsinos, Phys. Rev. D 96 (2017) 115008 [arXiv: 1708.09054] [INSPIRE].

[48] H. Baer, V. Barger, J.S. Gainer, D. Sengupta, H. Serce and X. Tata, LHC luminosity and energy upgrades confront natural supersymmetry models, Phys. Rev. D 98 (2018) 075010 [arXiv: 1808.04844] [INSPIRE].

[49] H.P. Nilles and P.K.S. Vaudrevange, Geography of Fields in Extra Dimensions: String Theory Lessons for Particle Physics, Adv. Ser. Direct. High Energy Phys. 22 (2015) 49 [InSPIRE].

[50] W. Buchmüller, K. Hamaguchi, O. Lebedev and M. Ratz, Local grand unification, in CP Violation and the Flavour Puzzle: Symposium in Honour of Gustavo C. Branco. GustavoFest 2005, Lisbon, Portugal, July 2005, pp. 143-156, 2005, hep-ph/0512326 [INSPIRE].

[51] ATLAS collaboration, Search for supersymmetry in final states with missing transverse momentum and multiple $b$-jets in proton-proton collisions at $\sqrt{s}=13 \mathrm{TeV}$ with the ATLAS detector, ATLAS-CONF-2018-041.

[52] CMS collaboration, Search for top squark pair production in pp collisions at $\sqrt{s}=13 \mathrm{TeV}$ using single lepton events, JHEP 10 (2017) 019 [arXiv:1706.04402] [INSPIRE].

[53] CMS collaboration, Search for additional neutral MSSM Higgs bosons in the $\tau \tau$ final state in proton-proton collisions at $\sqrt{s}=13 \mathrm{TeV}$, JHEP 09 (2018) 007 [arXiv: 1803. 06553] [INSPIRE].

[54] ATLAS collaboration, Search for electroweak production of supersymmetric states in scenarios with compressed mass spectra at $\sqrt{s}=13 \mathrm{TeV}$ with the ATLAS detector, Phys. Rev. D 97 (2018) 052010 [arXiv: 1712.08119] [INSPIRE]. 
[55] XENON collaboration, Dark Matter Search Results from a One Ton-Year Exposure of XENON1T, Phys. Rev. Lett. 121 (2018) 111302 [arXiv:1805.12562] [INSPIRE].

[56] H. Baer et al., Gluino reach and mass extraction at the LHC in radiatively-driven natural SUSY, Eur. Phys. J. C 77 (2017) 499 [arXiv: 1612.00795] [INSPIRE].

[57] H. Baer, V. Barger, M. Padeffke-Kirkland and X. Tata, Naturalness implies intra-generational degeneracy for decoupled squarks and sleptons, Phys. Rev. D 89 (2014) 037701 [arXiv: 1311.4587] [INSPIRE].

[58] H. Baer et al., Same sign diboson signature from supersymmetry models with light higgsinos at the LHC, Phys. Rev. Lett. 110 (2013) 151801 [arXiv:1302.5816] [InSPIRE].

[59] H. Baer et al., Radiatively-driven natural supersymmetry at the LHC, JHEP 12 (2013) 013 [Erratum ibid. 06 (2015) 053] [arXiv:1310.4858] [INSPIRE].

[60] H. Baer, V. Barger, J.S. Gainer, M. Savoy, D. Sengupta and X. Tata, Aspects of the same-sign diboson signature from wino pair production with light higgsinos at the high luminosity LHC, Phys. Rev. D 97 (2018) 035012 [arXiv:1710.09103] [InSPIRE].

[61] Z. Han, G.D. Kribs, A. Martin and A. Menon, Hunting quasidegenerate Higgsinos, Phys. Rev. D 89 (2014) 075007 [arXiv:1401.1235] [INSPIRE].

[62] H. Baer, A. Mustafayev and X. Tata, Monojet plus soft dilepton signal from light higgsino pair production at LHC14, Phys. Rev. D 90 (2014) 115007 [arXiv:1409.7058] [INSPIRE].

[63] C. Han, D. Kim, S. Munir and M. Park, Accessing the core of naturalness, nearly degenerate higgsinos, at the LHC, JHEP 04 (2015) 132 [arXiv:1502.03734] [INSPIRE].

[64] CMS collaboration, Search for new physics in events with two soft oppositely charged leptons and missing transverse momentum in proton-proton collisions at $\sqrt{s}=13 \mathrm{TeV}$, Phys. Lett. B 782 (2018) 440 [arXiv: 1801.01846] [INSPIRE].

[65] X. Cid Vidal et al., Beyond the Standard Model Physics at the HL-LHC and HE-LHC, arXiv:1812.07831 [INSPIRE].

[66] H. Baer, M. Bisset, C. Kao and X. Tata, Detecting Higgs boson decays to neutralinos at hadron supercolliders, Phys. Rev. D 50 (1994) 316 [hep-ph/9402265] [INSPIRE].

[67] K.J. Bae, H. Baer, N. Nagata and H. Serce, Prospects for Higgs coupling measurements in SUSY with radiatively-driven naturalness, Phys. Rev. D 92 (2015) 035006 [arXiv: 1505.03541] [INSPIRE].

[68] H. Baer, V. Barger and P. Huang, Hidden SUSY at the LHC: the light higgsino-world scenario and the role of a lepton collider, JHEP 11 (2011) 031 [arXiv:1107.5581] [INSPIRE].

[69] R. Allahverdi, B. Dutta and K. Sinha, Non-thermal Higgsino Dark Matter: Cosmological Motivations and Implications for a 125 GeV Higgs, Phys. Rev. D 86 (2012) 095016 [arXiv: 1208.0115] [INSPIRE].

[70] H. Baer, V. Barger and D. Mickelson, Direct and indirect detection of higgsino-like WIMPs: concluding the story of electroweak naturalness, Phys. Lett. B 726 (2013) 330 [arXiv: 1303.3816] [INSPIRE].

[71] K.J. Bae, H. Baer, V. Barger and D. Sengupta, Revisiting the SUSY $\mu$ problem and its solutions in the LHC era, arXiv:1902.10748 [INSPIRE].

[72] H. Baer, V. Barger, D. Sengupta and X. Tata, Is natural higgsino-only dark matter excluded?, Eur. Phys. J. C 78 (2018) 838 [arXiv:1803.11210] [InSPIRE]. 
[73] K.J. Bae, H. Baer, A. Lessa and H. Serce, Coupled Boltzmann computation of mixed axion neutralino dark matter in the SUSY DFSZ axion model, JCAP 10 (2014) 082 [arXiv: 1406.4138] [INSPIRE].

[74] XENON100 collaboration, XENON100 Dark Matter Results from a Combination of 477 Live Days, Phys. Rev. D 94 (2016) 122001 [arXiv:1609.06154] [INSPIRE].

[75] LUX-ZEPLIN collaboration, Projected WIMP Sensitivity of the LUX-ZEPLIN (LZ) Dark Matter Experiment, arXiv:1802.06039 [INSPIRE].

[76] G. Fiorillo, DarkSide-20k and the future Liquid Argon Dark Matter program, talk at UCLA DM Conference, Los Angeles, February 23, 2018 [https://indico.cern.ch/event/653314/ contributions/2825769/attachments/1606239/2548583/Fiorillo-DM2018.pdf].

[77] C. Galbiati, Direct Dark Matter Detection with Noble Gases, talk at UCLA DM Conference, Los Angeles, February 21, 2018 [https://indico.cern.ch/event/653314/contributions/ 2681549/attachments/1604699/2545488/Galbiati_UCLA_DM_2018_Feb_21_2018.pdf].

[78] PICO collaboration, Dark Matter Search Results from the PICO-60 $C_{3} F_{8}$ Bubble Chamber, Phys. Rev. Lett. 118 (2017) 251301 [arXiv:1702.07666] [INSPIRE].

[79] ICECUBE collaboration, Search for annihilating dark matter in the Sun with 3 years of IceCube data, Eur. Phys. J. C 77 (2017) 146 [Erratum ibid. C 79 (2019) 214] [arXiv: 1612.05949] [INSPIRE].

[80] MAGIC and Fermi-LAT collaborations, Limits to Dark Matter Annihilation Cross-Section from a Combined Analysis of MAGIC and Fermi-LAT Observations of Dwarf Satellite Galaxies, JCAP 02 (2016) 039 [arXiv:1601.06590] [INSPIRE].

[81] K.J. Bae, H. Baer and H. Serce, Prospects for axion detection in natural SUSY with mixed axion-higgsino dark matter: back to invisible?, JCAP 06 (2017) 024 [arXiv:1705.01134] [INSPIRE]. 